\title{
Comment faciliter la construction et la conduite des projets interdisciplinaires : retour d'expérience d'un programme de recherche sur la gestion intégrée de la santé des animaux en élevage
}

\author{
Christian Ducrot ${ }^{1, *}$, Maria Belen Barrio $^{2}$ (D), Alain Boissy ${ }^{3}$ (D), François Casabianca ${ }^{4}$ (D), \\ Marie-Hélène Pinard-van der Laan $^{5}$ (D) et Isabelle Maillet ${ }^{6}$ \\ 1 Épidémiologie, INRAE, UMR ASTRE, Montpellier, France \\ ${ }^{2}$ Immunologie, cheffe de projet du métaprogramme GISA, INRAE, UAR département Santé animale, Castanet-Tolosan, \\ France \\ ${ }^{3}$ Éthologie, INRAE, UMR Herbivores, Saint-Genès-Champanelle, France \\ 4 Zootechnie des systèmes d'élevage, INRAE, UMR SELMET-LRDE, Corte, France \\ 5 Génétique, INRAE, UMR GABI, Jouy-en-Josas, France \\ ${ }^{6}$ Microbiologie, coordinatrice des métaprogrammes, INRAE, UAR CODIR, Paris, France
}

\begin{abstract}
Résumé - L'article présente le retour d'expérience sur la conduite de l'interdisciplinarité dans 14 projets d'un programme sur la gestion intégrée de la santé des animaux. Les difficultés rencontrées portent sur l'acculturation et l'équilibre entre disciplines, l'animation scientifique et les publications. Le degré d'interdisciplinarité des projets va de la juxtaposition à l'intégration des disciplines. Les recommandations proposées aux porteurs de projet sont notamment le décentrage des questions disciplinaires pendant la phase de conception, une mise en œuvre basée sur une animation agile, et, pendant la phase de bilan, une analyse de l'apport de l'interdisciplinarité à la question initiale. Pour renforcer l'interdisciplinarité, le pilotage des programmes interdisciplinaires devrait donner la priorité à l'acculturation des chercheurs à l'interdisciplinarité, apporter un appui à la conception de projets et analyser le degré d'intégration des disciplines atteint dans les projets.
\end{abstract}

Mots-clés : agriculture / développement durable / interdisciplinarité / coordination de projets

\begin{abstract}
How to facilitate the design and management of interdisciplinary projects: feedback from a research program on integrated animal health management. The paper presents the feedback from the project leaders on the management of interdisciplinarity involving 14 scientific projects ( 3 networks and 11 research projects) that are part of a program on the integrated management of animal health, and from researchers participating in the overall management of the program. The difficulties encountered by the project leaders relate to a need for acculturation among disciplines, to the equilibrium and mutual respect between disciplines within the project, to the lack of time dedicated to the scientific animation of the project, and to the difficulty of publishing interdisciplinary work. The level of interdisciplinarity varies among projects, from juxtaposition of several disciplines to their actual integration. Recommendations proposed to future project leaders at the various steps of a projet involve the co-design step, in order to facilitate the decentering of disciplinary questioning, the implementation step requiring an agile management, and the report step, in order to analyse how interdisciplinarity has shifted disciplinary lines and modified initial questions. Strengthening of interdisciplinary programs should prioritize the training of researchers in interdisciplinarity, provide support for the conception of interdisciplinary projects, and analyse the degree to which integration of disciplines was achieved in the projects.
\end{abstract}

Keywords: agriculture / sustainable development / interdisciplinarity / project coordination

*Auteur correspondant : christian.ducrot@inrae.fr 


\section{Introduction}

La recherche s'est fortement développée pendant les $\mathrm{XIX}^{\mathrm{e}}$ et $\mathrm{XX}^{\mathrm{e}}$ siècles autour des disciplines, lesquelles se sont subdivisées au cours de l'avancée des sciences en de nouvelles disciplines encore plus spécialisées, parallèlement au développement des universités et des moyens octroyés à la recherche (Ledford, 2015). Cette approche disciplinaire, avec ses règles, son langage et ses méthodes propres a permis des avancées scientifiques considérables, mais apparaît inopérante pour aborder les questions particulièrement complexes. Les premières références à l'interdisciplinarité sont apparues dès 1937 (Ledford, 2015), puis, en 1994, un ouvrage (Gibbons et al., 1994) prédit le développement de l'interdisciplinarité pour aborder des questions socialement pertinentes. De nos jours, la réflexion interdisciplinaire est désormais un impératif en raison de la combinaison de quatre facteurs (National Academy of Sciences et al., 2005; National Research Council, 2014) : la complexité inhérente à la nature et à la société, la volonté d'explorer des problèmes qui dépassent le cadre d'une discipline, le besoin de résoudre des problèmes sociétaux et la puissance des nouvelles technologies. Les problèmes complexes ne peuvent pas être décomposés pour les étudier sans les dénaturer; l'interdisciplinarité permet alors de définir un objet commun et de l'analyser autrement et dans sa globalité en croisant les points de vue disciplinaires (Jollivet, 1992 ; Legay, 1988). La prévention des maladies est citée comme un des problèmes sociétaux complexes (National Academy of Sciences et al., 2005) et les enjeux autour de la santé sont très présents dans les travaux interdisciplinaires, que ce soit en santé humaine (Van Noorden, 2015) ou animale (Houe, 2003).

L'approche interdisciplinaire requiert une évolution nécessaire du cadre de travail dans les organisations de recherche (Billaud et Hubert, 2006; National Academy of Sciences et al., 2005), notamment en matière de formation à l'interdisciplinarité, de financement, de recrutement, d'évolution des parcours (individuels et collectifs) et d'évaluation des chercheurs et des projets (Hubert et Bonnemaire, 2000; National Academy of Sciences et al., 2005). Dans cette optique, l'Institut national de la recherche agronomique (Inra) a initié des «métaprogrammes» (MP) (Guillou et al., 2010). Transversaux aux approches disciplinaires et aux questions de recherche posées au sein des départements scientifiques de l'Inra, ils portent sur des thèmes stratégiques au regard des orientations de l'institut afin d'appréhender de façon coordonnée et intégrée les grands enjeux de la recherche agronomique, par exemple la gestion intégrée de la santé des animaux (GISA) ([n. s.], 2012). Les MP servent à identifier des défis, à les mettre en visibilité, à rendre plus attractif l'espace interdisciplinaire ([n.s.], 2015) et à stimuler le dialogue entre départements scientifiques afin d'aborder ces objets complexes de manière coordonnée et holistique.

Ces MP à l'Inra étaient une nouveauté, tant pour leurs coordinateurs que pour les porteurs de projet de recherche et, plus globalement, pour la communauté scientifique Inra. Même si dans l'ensemble, les chercheurs étaient convaincus du bien-fondé de l'approche, la plupart n'en avait que des expériences individuelles et fragmentaires. Les attentes en matière d'interdisciplinarité n'étaient pas approfondies dans le cadre proposé (Guillou et al., 2010); de ce fait, ces MP ont été le théâtre d'un apprentissage partagé de l'approche interdisciplinaire, à l'échelle des comités de pilotage (CoPil) des MP et des chercheurs ayant soumis des projets. L'approche interdisciplinaire a été mise en œuvre grâce aux instruments de financement accessibles, notamment des appels à manifestation d'intérêt (AMI).

Diverses publications abordent l'interdisciplinarité à l'échelle du chercheur, des programmes et des organisations (Brown et al., 2015; National Academy of Sciences et al., 2005 ; National Research Council, 2014 ; Pischke et al., 2017; de Pryck et al., 2018). En revanche, les publications qui abordent la pratique de l'interdisciplinarité à l'échelle du projet de recherche demeurent rares. Sur la base de l'expérience acquise en huit ans de pilotage du MP GISA et de l'analyse de 14 projets de recherche soutenus par ce MP, l'objet initial de ce texte ${ }^{1}$ était de présenter l'analyse du retour d'expérience de ces projets, conduite au sein du CoPil dudit MP, et de proposer des éléments et des méthodes de travail à même de faciliter la conception et la conduite de projets de recherche interdisciplinaires. Cette réflexion nous a amenés progressivement à nous interroger aussi sur le rôle du CoPil dans le renforcement de l'interdisciplinarité et, forts de l'analyse conduite, nous proposons quelques recommandations sur le pilotage d'un programme pour favoriser l'interdisciplinarité au sein des projets qu'il finance.

\section{Dispositif étudié : projets et réseaux financés au sein du métaprogramme Gestion intégrée de la santé des animaux}

Le MP GISA ([n.s.], 2012) (Fig. 1) a fonctionné de 2012 à 2019, animé par le chef du département Santé

\footnotetext{
${ }^{1}$ Le texte est basé sur le travail réflexif d'un collectif de chercheurs engagés dans le comité de pilotage du métaprogramme Inra sur la gestion intégrée de la santé des animaux, afin de capitaliser sur les retours d'expérience de 14 projets financés par ce métaprogramme, sur leur propre expérience dans la promotion et le suivi de l'interdisciplinarité dans ces projets, voire sur leur participation à certains d'entre eux.
} 
animale de l'Inra qui s'est appuyé sur un CoPil de 11 chercheurs de disciplines variées (microbiologie, immunologie, épidémiologie, nutrition, génétique, éthologie, zootechnie et systèmes d'élevage, économie et sociologie).

Six AMI ont été lancés pour financer des « réseaux » destinés à favoriser l'incubation de projets, ainsi que des projets de types «exploration» et «envergure» selon leur ambition, leur durée et leur niveau de prise de risque. Quel que soit le support choisi, le critère commun était de favoriser l'intégration des disciplines, et non leur seule juxtaposition, dans la définition et le traitement des questions de recherche. Dans les premiers AMI, il était mentionné comme critère d'éligibilité la nature multidisciplinaire du projet à travers l'articulation de plusieurs équipes et de plusieurs disciplines. À partir de 2014, les AMI demandaient de présenter les actions mises en place pour faciliter l'interdisciplinarité dans le projet. Enfin, à partir de 2015, comme certains projets soumis auparavant relevaient plutôt d'une addition de volets disciplinaires, l'AMI précisait que le terme d'interdisciplinarité signifiait une coopération étroite dépassant la simple juxtaposition entre disciplines scientifiques. Lors des rencontres bisannuelles du MP GISA, deux exposés ${ }^{2}$ ont été consacrés aux enjeux de l'interdisciplinarité (construction des disciplines pour l'un, transdisciplinarité pour l'autre), et un atelier a été organisé pour partager les expériences des chercheurs sur la pratique de l'interdisciplinarité. Le CoPil s'est appuyé sur un comité scientifique international qui avait, entre autres, à rendre compte d'expériences similaires conduites dans d'autres pays et à évaluer les projets sur la base d'une grille multicritères insistant tout particulièrement sur la complémentarité des partenaires relevant de différentes disciplines et sur la qualité des investigations. Il était composé de 12 scientifiques de renommée internationale travaillant dans des domaines disciplinaires variés (virologie, immunologie, épidémiologie, génétique, nutrition, éthologie, économie, sociologie), mais peu d'entre eux avaient une expérience de l'interdisciplinarité.

L'analyse a posteriori de la pratique effective de l'interdisciplinarité dans les projets financés dans le MP a été réalisée par les auteurs de l'article, cinq membres du CoPil et la coordinatrice des MP à l'Inra. Cette analyse a reposé sur trois étapes: 1) recueillir le retour d'expérience des porteurs de projet et de réseau sur leur pratique de l'interdisciplinarité, à partir d'un questionnaire

\footnotetext{
${ }^{2}$ En 2012, exposé de Terry Shinn (sociologue des sciences, CNRS): «Disciplines scientifiques: émergence, diffusion et disparition - Les régimes sociaux de production des connaissances »; en 2014, exposé de Basarab Nicolescu (physicien, Centre international de recherches et études transdisciplinaires): «Transdisciplinarité- Concept et déclinaisons ».
}

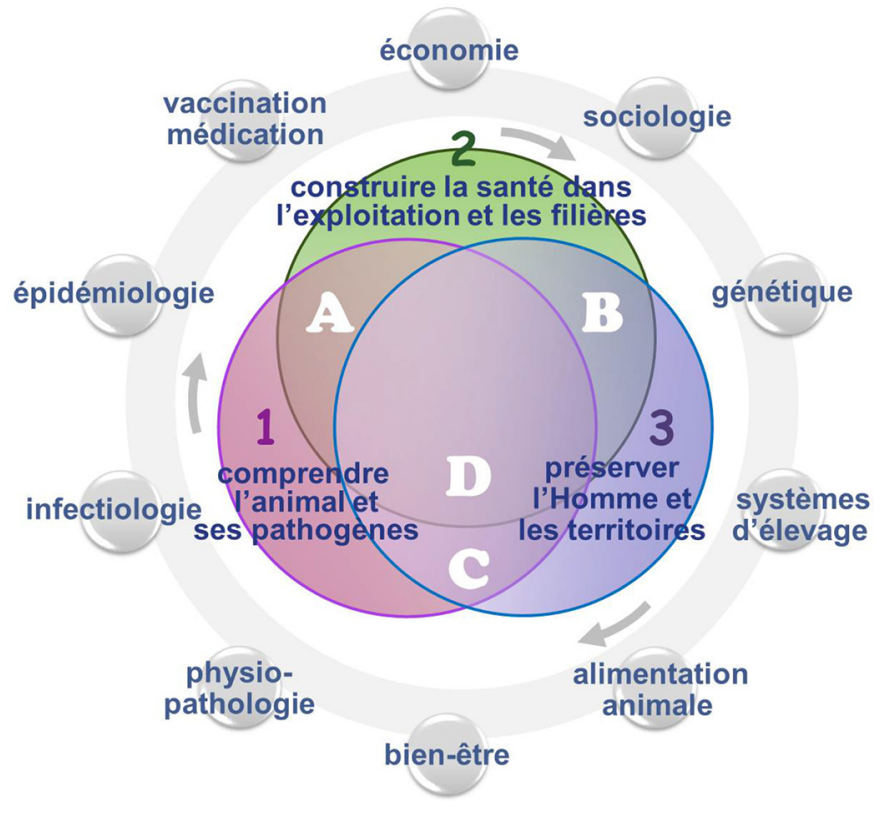

Fig. 1. Métaprogramme Gestion intégrée de la santé animale (GISA) : enjeux et disciplines impliquées. La santé est abordée à trois échelles complémentaires, de l'animal jusqu'au territoire en passant par la filière de production, et son étude repose sur le croisement de nombreuses disciplines des sciences animales et vétérinaires et des sciences humaines et sociales.

Source: Pineau Thierry, premières rencontres du métaprogramme GISA, 12-15 mars 2012.

qualitatif adressé à l'issue des projets ; 2) analyser les réponses au questionnaire (11 projets et 3 réseaux sur un total de 21 projets financés, voir Tab. 1) sur les difficultés rencontrées, le degré d'intégration des disciplines dans les projets et les démarches qui ont montré une certaine efficacité pour promouvoir l'interdisciplinarité; et 3 ) discuter des résultats obtenus.

Cette analyse a été enrichie par une réflexion sur ce qui pourrait être développé au niveau de l'animation d'un MP afin de faciliter la mise en œuvre de l'interdisciplinarité dans les projets de recherche qu'il finance.

\section{Résultats}

\section{Difficultés rencontrées par les porteurs de projet dans la mise en œuvre d'une recherche interdisciplinaire}

Dans leurs témoignages pris individuellement, les porteurs de projet ont fait part des mêmes difficultés dans la pratique de l'interdisciplinarité au sein de leurs projets ou réseaux, qui peuvent être regroupées en quatre thèmes.

i) Un besoin d'acculturation entre les communautés disciplinaires qui n'avait pas été suffisamment anticipé. Ce besoin, lié aux différences entre disciplines, concerne non seulement les concepts et le langage, mais aussi les 
Tab 1. Caractéristiques des 11 projets et 3 réseaux analysés, financés par le métaprogramme Gestion intégrée de la santé animale (GISA).

\begin{tabular}{|c|c|c|c|c|}
\hline Type & Acronyme & Thème du projet & $\begin{array}{l}\text { Nb départements } \\
+ \text { partenaires }\end{array}$ & $\begin{array}{l}\text { Expertises mobilisées au } \\
\text { sein de l'Inra }\end{array}$ \\
\hline Projet & EQUIBIO & $\begin{array}{l}\text { Maintien de l'équilibre: concept } \\
\text { et approche pour la gestion de } \\
\text { la santé et du bien-être en } \\
\text { élevage biologique de ruminants }\end{array}$ & $3+4$ & $\begin{array}{l}\text { Épidémiologie, éthologie, } \\
\text { nutrition, physiopathologie, } \\
\text { zootechnie et systèmes } \\
\text { d'élevage }\end{array}$ \\
\hline Projet & ROBUSTCHICK & $\begin{array}{l}\text { Impact de la température } \\
\text { d'incubation et de l'humidité } \\
\text { relative sur robustesse, santé et } \\
\text { bien-être des poulets ayant reçu } \\
\text { un challenge thermique }\end{array}$ & $4+3$ & $\begin{array}{l}\text { Éthologie, microbiologie, } \\
\text { physiopathologie, } \\
\text { zootechnie et systèmes } \\
\text { d'élevage }\end{array}$ \\
\hline Projet & TRAJ & $\begin{array}{l}\text { Trajectoires de changement } \\
\text { dans l'utilisation des } \\
\text { antimicrobiens en productions } \\
\text { animales }\end{array}$ & $2+5$ & $\begin{array}{l}\text { Économie, épidémiologie, } \\
\text { physiopathologie, science de } \\
\text { la communication, } \\
\text { sociologie }\end{array}$ \\
\hline Projet & COPPECS & $\begin{array}{l}\text { Corégulation des services de } \\
\text { conseil publics et privés et } \\
\text { standards pour la gestion de la } \\
\text { santé animale }\end{array}$ & $3+1$ & $\begin{array}{l}\text { Économie, physiopathologie, } \\
\text { sociologie }\end{array}$ \\
\hline Projet & RUMINFLAME & $\begin{array}{l}\text { Amélioration du diagnostic des } \\
\text { maladies inflammatoires et de la } \\
\text { prédiction du statut } \\
\text { inflammatoire local et } \\
\text { systémique des ruminants laitiers }\end{array}$ & $4+5$ & $\begin{array}{l}\text { Économie, génétique, } \\
\text { immunologie, infectiologie, } \\
\text { microbiologie, nutrition, } \\
\text { physiologie, } \\
\text { physiopathologie, zootechnie } \\
\text { et systèmes d'élevage }\end{array}$ \\
\hline Projet & PACMAN & $\begin{array}{l}\text { Coordination du pathosystème, } \\
\text { management des réseaux } \\
\text { humain et animal }\end{array}$ & $3+10$ & $\begin{array}{l}\text { Épidémiologie, management } \\
\text { des sciences, sociologie, } \\
\text { zootechnie et systèmes } \\
\text { d'élevage }\end{array}$ \\
\hline Projet & HEALTHYGROWTH & $\begin{array}{l}\text { Exploration de la compétition } \\
\text { entre santé et croissance des } \\
\text { porcs }\end{array}$ & $2+3$ & $\begin{array}{l}\text { Éthologie, génétique, } \\
\text { informatique, } \\
\text { physiopathologie }\end{array}$ \\
\hline Projet & WHELP & $\begin{array}{l}\text { Plateforme pour le bien-être et } \\
\text { la santé en période néonatale }\end{array}$ & $4+7$ & $\begin{array}{l}\text { Épidémiologie, éthologie, } \\
\text { génétique, immunologie, } \\
\text { nutrition, pharmacologie, } \\
\text { physiopathologie, zootechnie } \\
\text { et systèmes d'élevage }\end{array}$ \\
\hline Projet & eFEDBIOTA & $\begin{array}{l}\text { Modalité d'alimentation précoce } \\
\text { des lapereaux pour piloter le } \\
\text { microbiote digestif vers un } \\
\text { renforcement du système } \\
\text { immunitaire }\end{array}$ & $2+3$ & $\begin{array}{l}\text { Écologie microbienne, } \\
\text { immunologie, nutrition, } \\
\text { physiopathologie, } \\
\text { zootechnie et systèmes } \\
\text { d'élevage }\end{array}$ \\
\hline Projet & GALMIDE & $\begin{array}{l}\text { Vers un poulet plus robuste: } \\
\text { étude intégrée de l'efficacité } \\
\text { digestive, du statut immunitaire et } \\
\text { de la composition du microbiote }\end{array}$ & $3+0$ & $\begin{array}{l}\text { Génétique, immunologie, } \\
\text { nutrition, physiopathologie }\end{array}$ \\
\hline
\end{tabular}


Tab 1. (suite).

\begin{tabular}{|c|c|c|c|c|}
\hline Type & Acronyme & Thème du projet & $\begin{array}{l}\text { Nb départements } \\
+ \text { partenaires }\end{array}$ & $\begin{array}{l}\text { Expertises mobilisées au } \\
\text { sein de l'Inra }\end{array}$ \\
\hline Projet & AHOL & $\begin{array}{l}\text { Outil d'ontologie pour } \\
\text { l'intégration de la } \\
\text { multiperformance au niveau de } \\
\text { l'animal et du système dans le } \\
\text { secteur de l'élevage }\end{array}$ & $4+1$ & $\begin{array}{l}\text { Bio-informatique, } \\
\text { épidémiologie, éthologie, } \\
\text { génétique, immunologie, } \\
\text { microbiologie, nutrition, } \\
\text { pharmacologie, } \\
\text { physiopathologie, zootechnie } \\
\text { et systèmes d'élevage }\end{array}$ \\
\hline Réseau & $\mathrm{R} 2 \mathrm{~A} 2$ & $\begin{array}{l}\text { Réseau de recherche sur la } \\
\text { réduction d'usage des } \\
\text { antibiotiques et la résistance aux } \\
\text { antibiotiques en élevage }\end{array}$ & $10+31$ & $\begin{array}{l}\text { Toutes les disciplines des } \\
\text { sciences animales et } \\
\text { vétérinaires, économie, } \\
\text { sociologie }\end{array}$ \\
\hline Réseau & SAEB & $\begin{array}{l}\text { Gestion de la santé en élevage } \\
\text { biologique }\end{array}$ & $4+8$ & $\begin{array}{l}\text { Économie, épidémiologie, } \\
\text { éthologie, microbiologie, } \\
\text { nutrition, physiopathologie, } \\
\text { sociologie, zootechnie et } \\
\text { systèmes d'élevage }\end{array}$ \\
\hline
\end{tabular}

* Nombre de départements scientifiques Inra impliqués + nombre de partenaires académiques et socioprofessionnels

méthodes, les données, les types de résultats obtenus et leur interprétation. Certains verbatims extraits des questionnaires illustrent ce point :

«Les seules barrières ont été celles liées au fait de s'aventurer sur les territoires de disciplines qu'on ne pratique pas, i.e. difficultés avec la terminologie, les concepts et les méthodes. Les présentations et les temps de discussion informelle ont été très utiles pour lever ces barrières. »)

«Il a été difficile d'anticiper les problèmes pratiques liés au manque de connaissance/pratique avec les outils des autres disciplines engagées. L'interdisciplinarité requiert l'appropriation de nouvelles connaissances, de méthodes, de façons de penser; c'est de ce fait déstabilisant et prend énormément de temps. »

ii) L'équilibre et le respect mutuel entre disciplines dans le montage et le pilotage du projet. L'écueil principal est l'instrumentalisation d'une discipline par une autre, mais aussi le défaut de coconstruction en amont.

«Ça ne marche pas si le projet est celui d'un des partenaires, utilisant les compétences des autres. Dans ce cas, le responsable du projet devrait faire appel à des ingénieurs plutôt qu'à des chercheurs. »)

«Dans le projet, nous (sociologues) avons réalisé que nous n'avions pas collecté les données dont les chercheurs en sciences animales et vétérinaires auraient eu besoin pour contribuer plus à l'analyse des résultats. »

iii) Les exigences spécifiques aux projets interdisciplinaires. Les modalités de coconstruction en amont, d'animation scientifique pendant le projet, et le temps nécessaire pour sa mise en œuvre sont souvent sous-estimés.

«Chaque partenaire est venu avec ses questions spécifiques, et nous nous sommes retrouvés avec plein de questions déconnectées les unes des autres sur la table... Nous nous sommes retrouvés avec les partenaires du projet devant gérer à la fois les tâches du projet (décidées collectivement) et leur programme initial. »

«Un jour de rencontre par an pour le projet n'était pas suffisant. »

iv) La difficulté de valoriser les travaux interdisciplinaires, étant donné le nombre restreint de revues adaptées à l'interdisciplinarité. Cela est couplé aux différences de culture scientifique selon les domaines.

«Un autre point a été de définir une stratégie de publication, car il n'y a pas tant d'espaces académiques interdisciplinaires. Nous avons finalement opté pour des journaux et conférences vétérinaires et de sciences animales ouverts au social. »

«Il y a peu de journaux publiant des travaux interdisciplinaires. Il est par exemple difficile de publier des travaux 
qualitatifs dans une revue de sciences animales. Les sciences animales requièrent plus de données pour atteindre un niveau suffisant de généricité. »

\section{Degré d'intégration des disciplines dans les projets}

Les commentaires des porteurs de projet et l'analyse de la structure des projets et des questions posées montrent des différences quant au degré d'interdisciplinarité atteint.

D'un côté, il y a des projets avec les tâches organisées par discipline, chacune abordant une question disciplinaire relative au thème du projet. Ce type de projet s'apparente plus à la pluridisciplinarité, chaque discipline amenant sa contribution pour résoudre une partie de la question.

«Le projet a été subdivisé par discipline pour faciliter le travail de chaque chercheur; seul un volet était interdisciplinaire, pour intégrer les résultats. Cette organisation n'a pas été optimale pour aborder la gestion de la santé d'un point de vue interdisciplinaire. En conséquence, chaque chercheur s'est concentré sur ses propres données, ce qui a résulté en une faible avancée du travail prévu.»

De l'autre, il y a des projets avec des tâches organisées en fonction de questions finalisées, plusieurs disciplines étant impliquées dans chaque tâche pour y répondre, avec donc une réelle tentative d'approche interdisciplinaire.

«Le degré d'interdisciplinarité a été permis par le fait de travailler sur les mêmes jeux de données et de ne pas séparer les tâches par discipline. »

\section{Discussion}

La discussion aborde successivement les recommandations pertinentes pour la conduite de l'interdisciplinarité au sein des projets, et les enseignements que nous avons tirés de notre expérience pour qu'un programme facilite le cheminement des projets vers l'interdisciplinarité.

\section{Recommandations pour la conduite de projets interdisciplinaires}

Selon les retours d'expérience des porteurs de projet, les difficultés rencontrées dans la conduite des projets interdisciplinaires sont très largement partagées avec celles décrites dans la littérature (National Academy of Sciences et al., 2005; National Research Council, 2014). Plusieurs recommandations peuvent être proposées afin de stimuler l'interdisciplinarité à l'échelle d'un projet de recherche; elles sont présentées ici selon trois étapes-clés.

\section{Conception du projet}

Le temps consacré à la conception du projet est généralement sous-estimé. C'est pourtant une étape essentielle pour positionner le projet dans un cadre favorable à l'interdisciplinarité. L'enjeu majeur est de décentrer les points de vue disciplinaires. Cette phase est le moment privilégié pour poser les questions auxquelles le collectif pluridisciplinaire entend répondre, et qui ne sont pas seulement celles des chercheurs pris individuellement. Pour faciliter ce cheminement vers un questionnement partagé, l'analyse montre que le fait d'avoir déjà échangé avec les chercheurs d'autres disciplines est un atout très important qui permet une acculturation aux autres disciplines et un gain de temps lors de la conception de nouveaux projets. De plus, à ce stade, il est essentiel de participer avec un état d'esprit ouvert aux autres disciplines, comme le souligne Barrué-Pastor (1992). Les discussions au sein du réseau SAEB ont par exemple été un atout fort pour la conception et la mise en œuvre du projet EQUIBIO.

Une approche de type «problem oriented research» (Katona et Curtin, 1980), mise en œuvre dans le projet PACMAN, facilite ce cheminement intellectuel car elle part des questions concrètes du terrain et conduit à réfléchir à la façon d'associer les compétences disciplinaires pour y répondre ensemble. Elle amène le chercheur à dépasser son questionnement disciplinaire initial pour concevoir, avec les chercheurs d'autres disciplines, la solution du problème. Néanmoins, un point délicat est de réaliser ce cheminement tout en gardant son ancrage disciplinaire, ce que nous ne développerons pas dans cet article.

La mise en œuvre d'une approche participative, en intégrant les porteurs d'enjeux dès la conception du projet, peut faciliter l'interdisciplinarité ${ }^{3}$ dans le sens où elle amène à s'intéresser à leurs questionnements, par essence non disciplinaires ; le projet HEALTHYGROWTH a mené une concertation de ce type en tout début de processus pour comprendre les attentes des acteurs de terrain. Les chercheurs sont ainsi amenés à s'extraire de leur zone de confort disciplinaire. Associer les parties prenantes au choix des hypothèses de travail peut sembler difficile et nécessiter plus de temps. Néanmoins, cette coconception enrichit le projet par les réflexions pertinentes des acteurs de terrain sur les questions qu'ils se posent dans leur métier (Calavas et Rosner, 1997 ; Deffontaines et Hubert, 2004). Le travail de traduction des questions de société en questions de recherche est en soi un processus long et complexe, qui nécessite du temps (Hubert et Bonnemaire, 2000; Houe,

\footnotetext{
${ }^{3}$ L'intégration des parties prenantes à la conception du projet relève plus de transdisciplinarité que d'interdisciplinarité, mais ce n'est pas le propos de l'article de le développer.
} 
2003). Timmermans et al. (2018) proposent d'intégrer dans le cadre de la construction des projets interdisciplinaires une phase de reformulation des problèmes afin de faciliter la compréhension mutuelle.

Ces recommandations rejoignent certaines préoccupations formulées dans la littérature. La proposition de "problem oriented research» sous-entend un travail en commun entre les chercheurs de disciplines différentes et les parties prenantes. Ce partage représente une bonne base pour la mise en place, à la fin des travaux, de recommandations sur l'impact sociétal (Brown et al., 2015), et pour la conception de programmes communs entre organismes scientifiques, entreprises, organisations professionnelles publiques ou privées et associations (National Academy of Sciences et al., 2005). La volonté de travailler avec les parties prenantes implique l'utilisation d'un langage sans jargon scientifique et une forme d'empathie et de respect mutuel, propices à l'interdisciplinarité (Brown et al., 2015), de même que la nécessité de consacrer suffisamment de temps à ces travaux (Houe, 2003; [n.s.], 2015). Cette approche peut mobiliser la conception innovante ${ }^{4}$ (Hatchuel et al., 2017), ce qui a été le cas au sein du réseau R2A2 sur les questions posées par l'usage des antibiotiques en élevage (Vourc'h et al., 2018) et a grandement facilité le travail collectif. Elle a permis de rediscuter le statut des connaissances et de concevoir une situation de recherche qui n'était plus celle de départ, décentrant la réflexion disciplinaire (Ledford, 2015; Brown et al., 2015).

À cette étape, deux stratégies positives ont été relevées dans les retours d'expérience des porteurs de projet. La première est de concevoir le projet sous forme de parties elles-mêmes interdisciplinaires, et de mettre en place une tâche de suivi de l'interdisciplinarité du projet par une personne extérieure, familiarisée avec les travaux interdisciplinaires. Ce suivi peut mettre en valeur ce que l'interdisciplinarité produit de nouveau, d'intellectuellement stimulant, sur la base des retours positifs sur le collectif et des parcours individuels des chercheurs; il peut permettre de s'assurer de la reconnaissance des différentes disciplines embarquées. La seconde stratégie, qui s'inscrit dans la durée, consiste à prévoir dans le cadre du projet des thèses coencadrées par des chercheurs de disciplines différentes (National Academy of Sciences et al., 2005 ; Timmermans et al., 2018) afin de permettre la formation de chercheurs aux interfaces, de structurer une collaboration longue entre encadrants de disciplines

\footnotetext{
${ }^{4}$ La conception innovante et les travaux théoriques qui la soustendent ont permis de mettre en place des méthodologies de l'innovation pour l'entreprise. Ces méthodes ont pour vocation de «défixer» les gens. Elles permettent aussi aux différents métiers (ingénieurs, designers, chercheurs...) de travailler ensemble et les poussent à explorer l'inconnu de façon systématique et collective.
}

différentes et, ainsi, de favoriser un travail interdisciplinaire en profondeur. Ces deux approches ont été combinées au sein du projet TRAJ, dans une interface entre sociologie et épidémiologie pour étudier l'usage des antibiotiques en élevage.

\section{Mise en œuvre et suivi du projet}

La mise en œuvre d'un projet interdisciplinaire requiert de prévoir beaucoup de temps pour échanger, de préférence en présentiel, et ainsi se familiariser avec les concepts, la terminologie et les méthodes des autres disciplines. Presque tous les porteurs de projet l'ont mentionné mais, une fois de plus, ce temps de sensibilisation au raisonnement et au fonctionnement des autres disciplines est sous-estimé (Ledford, 2015).

Le maître-mot pour le suivi du projet est une animation réactive, au sens où le déroulé est difficilement prévisible car le cheminement de la recherche interdisciplinaire est encore moins linéaire que celui de la recherche disciplinaire. La réactivité dans l'adaptation de l'objet de recherche, l'animation du projet, alliée à une certaine flexibilité dans l'organisation et le déroulement des tâches sont des atouts décisifs. Cette réactivité est particulièrement importante pour la reconnaissance de chacune des disciplines présentes. Il peut en effet y avoir des dérives sur les équilibres entre disciplines, notamment l'instrumentalisation de l'une au profit de l'autre (cf. exemples cités par Deffontaines et Hubert [2004]). L'équilibre entre coordination et appui, entre contribution et bénéfice pour chaque discipline, est fragile (National Academy of Sciences et al., 2005) et nécessite d'en discuter ouvertement. Par ailleurs, Ledford (2015), ainsi que Brown et al. (2015) et Urbanska et al. (2019), pointent des déséquilibres de notoriété entre disciplines, les sciences humaines étant vues de façon condescendante par les sciences naturelles ou biophysiques; de tels déséquilibres ne permettent pas de bâtir des relations durables (Ledford, 2015).

Maintenir la motivation et la dynamique de collaboration interdisciplinaire est le meilleur moyen d'éviter ces dérives, ce qui impose d'établir un mode d'animation adapté au projet. La communication et le temps d'échange en cours de projet (Houe, 2003) ainsi que le leadership (National Academy of Sciences et al., 2005) sont déterminants pour la réussite de l'interdisciplinarité. Certains porteurs de projet font état du rôle positif d'un chercheur non spécialiste impliqué dans le projet et qui a fait office de médiateur pour favoriser les échanges, ou du rôle positif d'une discipline intégratrice ou «charnière» qui a facilité le travail entre disciplines.

\section{Bilan et évaluation}

En fin de projet, une phase de réflexivité et d'analyse rétrospective est utile pour tirer les enseignements du 
projet et améliorer ultérieurement la conduite de l'interdisciplinarité. Elle peut prendre différentes formes et permet de reformuler le problème initial en fonction des résultats obtenus, de discuter du ressenti des chercheurs des différentes disciplines autant que des difficultés rencontrées et des solutions apportées. En matière d'interdisciplinarité, cette réflexion conduit à analyser dans quelle mesure la conception du projet a favorisé des avancées significatives pour traiter le problème identifié alors que chaque discipline prise isolément n'aurait pas pu le faire. L'analyse rétrospective permet également d'alimenter en retour la réflexion de chacune des disciplines impliquées dans le projet. Ce retour de l'interdisciplinarité aux disciplines, élément majeur de cette phase d'évaluation (Barrué-Pastor, 1992) donne l'occasion de voir comment le détour par l'interdisciplinarité a enrichi, ou pas, les avancées des disciplines.

\section{Principes d'animation d'un programme basé sur l'interdisciplinarité}

Par manque d'expérience du CoPil du MP GISA, la question de la conduite de l'interdisciplinarité n'avait pas été mise en avant dans l'animation du collectif. L'analyse des retours d'expérience des porteurs de projet et l'expérience de coordination du MP permettent de proposer désormais quelques principes pour favoriser le développement de l'interdisciplinarité dans le cadre d'un programme de recherche tel qu'un MP.

\section{Facilitation des échanges entre disciplines}

La mise en œuvre de l'interdisciplinarité nécessite d'instaurer des échanges entre participants afin de croiser leurs regards. Lors du démarrage du programme, il est important que le CoPil explicite, à un large public de chercheurs de disciplines différentes, sa vision du périmètre thématique, et partage sa compréhension de l'interdisciplinarité et des concepts, pour en permettre une meilleure appropriation par les participants. Ce moment fondateur permettra d'initier un questionnement commun autour d'objets complexes et d'aborder les recherches afférentes avec un langage partagé.

D'autres moments d'échanges sont indispensables pour renforcer une compréhension commune des enjeux du programme, s'approprier les évolutions issues de l'avancée des projets de recherche et accompagner les nouveaux questionnements. La mise en place d'ateliers dédiés à l'enjeu interdisciplinaire peut contribuer au cheminement réflexif entre la communauté scientifique et le CoPil. C'est l'esprit dans lequel le GIS Climat a repensé son fonctionnement à partir de 2009 (de Pryck et al., 2018), faisant de l'animation scientifique au sein du programme un «lieu de rencontre» pour faire émerger de nouvelles idées et motiver des initiatives interdisciplinaires.

\section{Aide à la construction et à la conduite des projets}

Il est important que les AMI dans le cadre du programme soient le plus explicite possible quant aux attentes sur l'interdisciplinarité, et que ces attentes soient bien comprises par les futurs porteurs de projet. Par ailleurs, un processus impliquant le CoPil dans le conseil au montage de projet est recommandé. En effet, compte tenu de son travail de concertation en amont et de l'expérience acquise tout le long du programme, le CoPil est «garant» de la mise en place et de la pratique de l'interdisciplinarité dans les projets. Ses membres constituent des personnes référentes dont un des rôles est d'interagir régulièrement avec les équipes des projets pour accompagner la mise en œuvre de l'interdisciplinarité. Ces référents sont alors en mesure de pointer les risques liés à une méconnaissance de l'interdisciplinarité et d'intervenir pour aider à surmonter les difficultés. De même, ils sont en mesure de partager avec l'ensemble du CoPil des questionnements et réflexions non encore explorées. C'est pour une part ce qu'a mis en œuvre le GIS Climat à partir de 2010 (de Pryck et al., 2018), en instaurant un dialogue entre les porteurs de projet et les membres du comité d'orientation, dans un cadre de coconstruction.

\section{Évaluation du degré d'intégration des disciplines dans les projets}

L'expérience acquise dans la conduite du MP GISA et l'analyse du retour d'expérience des porteurs de projet conduisent à définir des critères pour évaluer le degré d'intégration des disciplines d'un projet et les chances de réussite de la démarche proposée. Parmi l'ensemble des recommandations détaillées précédemment, quatre critères-clés sont à mettre en exergue: i) le degré de complexité de la question initiale, ii) sa reformulation avec le processus de décentration des questions disciplinaires qui en résulte, iii) le type de structuration du projet mêlant les disciplines dans des parties de projet communes et prévoyant un pilotage agile de l'interdisciplinarité, et iv) l'anticipation des difficultés liées à l'interdisciplinarité (temps nécessaire, compréhension réciproque et positionnement de chacune des disciplines).

\section{Adaptation des $A M I$ et du financement}

Comme nous l'avons exposé tout au long de cet article, construire une interdisciplinarité prend du temps ([n.s.], 2015) et le cheminement de la recherche interdisciplinaire est beaucoup plus incertain que celui des projets disciplinaires. Le type d'AMI et le mode de financement doivent tenir compte du degré important d'incertitude quant à la durée et l'évolution du projet. Gleed et Marchant (2016) et Timmermans et al. (2018) plaident pour l'adaptation des modes d'évaluation des 
projets et des durées de financement. Citons là encore le GIS Climat qui a expérimenté avec succès (de Pryck et al., 2018) la flexibilité des appels à projets (au fil de l'eau et sous différentes formes), l'incubation de projets sur un an et la coconstruction des projets avec les membres du comité d'orientation, parallèlement à un fonctionnement souple de ce comité.

\section{Conclusion}

L'approche interdisciplinaire d'un objet de recherche complexe est à construire en s'appuyant sur des compétences disciplinaires complémentaires. Dans cette construction, la réflexion que nous avons conduite, abordant la conduite des projets interdisciplinaires et le pilotage d'un programme qui héberge ces projets, a permis de proposer des recommandations aux futurs porteurs de ces projets et des principes d'animation de programmes basés sur l'interdisciplinarité. Cette réflexion est actuellement intégrée dans le pilotage d'un nouveau MP portant sur la santé et le bien-être des animaux en élevage. Des présentations sur l'interdisciplinarité ont ainsi été faites lors du séminaire de lancement de ce programme. À l'issue de la sélection des lettres d'intention en réponse à l'AMI, un retour a été formalisé vers les porteurs de projet, notamment sur la construction de l'interdisciplinarité. Enfin, une coconstruction entre le CoPil et des acteurs volontaires de la communauté scientifique est en cours de réflexion pour l'élaboration de plus gros projets et leur suivi dans le temps. La durée des financements reste quant à elle contrainte par le cadre imposé.

Par ailleurs, la conduite de l'interdisciplinarité ne peut être efficace que dans un contexte adapté quant aux aspects formation, reconnaissance et financement (Bark et al., 2016). Un séminaire récent sur l'interdisciplinarité tenu à INRAE a largement insisté sur ces éléments, pointant des améliorations souhaitables. Après huit ans de fonctionnement et une évaluation positive des MP à l'Inra, des évolutions du dispositif sont envisagées dans le cadre de la nouvelle génération de MP. Ces évolutions, basées sur l'expérience acquise, ont pour objectif de permettre au plus grand nombre de scientifiques de se familiariser aux défis de l'interdisciplinarité ([n.s.], 2019). Outre l'organisation de séminaires, le CoPil sera notamment amené à mobiliser des outils de conception innovante telle la théorie $\mathrm{C}-\mathrm{K}$, pour développer une animation interdisciplinaire et soutenir la créativité, ainsi que des méthodologies du type ASIRPA-RT (Joly et al., 2019) pour mieux intégrer les attentes des acteurs et

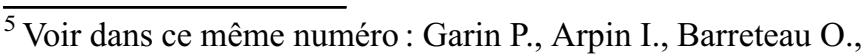
Caranta C., Ducrot C., Hannachi M., Maillet I., 2021. Réfléchir l'interdisciplinarité à INRAE, Natures Sciences Sociétés, 29, 2.
}

augmenter de la sorte l'impact des travaux de recherche interdisciplinaires.

\section{Remerciements}

Les auteurs remercient les autres membres du CoPil du MP GISA ainsi que les porteurs des projets soutenus, qui ont contribué à la réflexion collective en acceptant de faire part des difficultés rencontrées et des approches mises en œuvre pour conduire l'interdisciplinarité dans leurs projets.

\section{Références}

[n.s.], 2012. Appel à manifestation d'intérêt (AMI) du métaprogramme GISA, Paris, Inra.

[n.s.], 2015. Mind meld. Interdisciplinary science must break down barriers between fields to build common ground, Nature, 525, 289-290, https://doi.org/10.1038/525289b.

[n.s.], 2019. Rapport d'activité Inra 2018. Rapport, Paris, Inra.

Bark R.H., Kragt M.E., Robson B.J., 2016. Evaluating an interdisciplinary research project: lessons learned for organisation, researchers and funders, International Journal of Project Management, 34, 1449-1459, https://doi.org/ 10.1016/j.ijproman.2016.08.004.

Barrué-Pastor M., 1992. L'interdisciplinarité en pratiques, in Jollivet M. (Ed.), Sciences de la nature, sciences de la société. Les passeurs de frontières, Paris, CNRS Éditions, 365-380, https://doi.org/10.4000/books.editionscnrs.4211.

Billaud J.-P., Hubert B., 2006. Interdisciplinarité et production de connaissances: quelles recherches voulons-nous?, Natures Sciences Sociétés, 14, 233-234, https://doi.org/ 10.1051/nss:2006033.

Brown R.R., Deletic A., Wong T.H.F., 2015. Interdisciplinarity: how to catalyse collaboration, Nature, 525, 315-317, https://doi.org/10.1038/525315a.

Calavas D., Rosner G., 1997. Institutionalisation d'une recherche-action en santé animale : l'expérience du Centre d'écopathologie animale, Études et recherches sur les systèmes agraires et le développement, 30, 91-113, $<$ hal-02841713>.

Deffontaines J.-P., Hubert B., 2004. Un regard sur l'interdisciplinarité à l'Inra. Point de vue de deux chercheurs du département Sciences pour l'action et le développement (SAD), Natures Sciences Sociétés, 12, 186-190, https://doi. org/10.1051/nss:2004025.

De Pryck K., Vanderlinden J.-P., Billaud J.-P., 2018. L'animation scientifique dans la pratique interdisciplinaire : l'expérience du GIS Climat Environnement Société (2007-2015), Natures Sciences Sociétés, 26, 1, 76-83, https://doi.org/10.1051/nss/2018025.

Gibbons M., Limoges C., Nowotny H., Schwartzman S., Scott P., Trow M., 1994. The new production of knowledge. The dynamics of science and research in contemporary societies, London, Sage Publications.

Gleed A., Marchant D., 2016. Interdisciplinarity. Survey report for the Global Research Council, DJS Research, 
https://www.globalresearchcouncil.org/fileadmin/docu ments/GRC_Publications/Interdisciplinarity_Report_ for_GRC_DJS_Research.pdf.

Guillou M., Ribā G., Houllier F., Eddi M., Leverve X., Guyomard H., Soussana J.-F., Chemineau P., 2010. Document d'orientation Inra 2010-2020. Une science pour l'impact. Rapport, Paris, Inra, https://hal.inrae.fr/hal02823708.

Hatchuel A., le Masson P., Weil B., 2017. C-K theory: modelling creativity thinking and its impact on research, in Darbellay F., Moody Z., Lubart T. (Eds), Creativity, design thinking and interdisciplinarity. Creativity in the twenty first century, Springer, 169-181.

Houe H., 2003. Co-ordinated interdisciplinary efforts on research in animal production and health, Acta Veterinaria Scandinavica, 44, S51, https://doi.org/10.1186/1751-014744-S1-S51.

Hubert B., Bonnemaire J., 2000. La construction des objets dans la recherche interdisciplinaire finalisée, Natures Sciences Sociétés, 8, 3, 5-19, https://doi.org/10.1016/ S1240-1307(00)80056-4.

Jollivet M. (Ed.), 1992. Sciences de la nature, sciences de la société. Les passeurs de frontières, Paris, CNRS Éditions, https://doi.org/10.4000/books.editionscnrs.4154.

Joly P.-B., Matt M., Robinson D.K.R., 2019. Research impact assessment: from ex post to realtime assessment, Journal for Research and technology Policy Evaluation, 47, 35-40, https://doi.org/10.22163/fteval.2019.326.

Katona G., Curtin R.T., 1980. Problem-oriented rather than discipline-oriented research, in Olson J.C. (Ed.), NA Advances in Consumer Research, Ann Arbor, Association for Consumer Research, 44-45, https:/www.acrwebsite. org/volumes/9645/.

Ledford H., 2015. How to solve the world's biggest problems, Nature, 525, 308-311, https://doi.org/10.1038/525308a.
Legay J.-M., 1988. Méthodes et modèles dans l'étude des systèmes complexes, in Jollivet M. (Ed.), Pour une agriculture diversifiée, Paris, L'Harmattan, 14-22.

National Academy of Sciences, National Academy of Engineering, Institute of Medicine, 2005. Facilitating interdisciplinary research, Washington, The National Academies Press, https://doi.org/10.17226/11153.

National Research Council, 2014. Convergence. Facilitating transdisciplinary integration of life sciences, physical sciences, engineering, and beyond, Washington, The National Academies Press, https://doi.org/10.17226/18722.

Pischke E.C., Knowlton J.L., Phifer C.C., Gutierrez Lopez J., Propato T.S., Eastmond A., de Souza T.M., Kuhlberg M., Picasso Risso V., Veron S.R., Garcia C., Chiappe M., Halvorsen K.E., 2017. Barriers and solutions to conducting large international, interdisciplinary research projects, Environnemental Management, 60, 6, 1011-1021, https:// doi.org/10.1007/s00267-017-0939-8.

Timmermans B., Baret P., Hiernaux Q., Lugen M., Nonclercq A., Zaccai E., 2018. L'interdisciplinarité, ça marche! Une enquête et un colloque révèlent des facteurs de succès, Natures Sciences Sociétés, 26, 1, 67-75, https://doi.org/ $10.1051 / \mathrm{nss} / 2018023$.

Urbanska K., Huet S., Guimond S., 2019. Does increased interdisciplinary contact among hard and social scientists help or hinder interdisciplinary research?, PLoS One, 14, 9, e0221907, https://doi.org/10.1371/journal.pone.0221907.

Van Noorden R., 2015. Interdisciplinary research by the numbers, Nature, 525, 306-307, https://doi.org/10.1038/525306a.

Vourc'h G., Brun J., Ducrot C., Cosson J.-F., le Masson P., Weil B., 2018. Using design theory to foster innovative crossdisciplinary research: lessons learned from a research network focused on antimicrobial use and animal microbes' resistance to antimicrobials, Veterinary and Animal Science, 6, 12-20, https://doi.org/10.1016/j.vas.2018.04.001.

Citation de l'article : Ducrot C., Barrio M.B., Boissy A., Casabianca F., Pinard-van der Laan M.-H., Maillet I. Comment faciliter la construction et la conduite des projets interdisciplinaires : retour d'expérience d'un programme de recherche sur la gestion intégrée de la santé des animaux en élevage. Nat. Sci. Soc. 29, 2, 213-222. 